\title{
IMPLANTACIÓN DE UN SISTEMA DE GESTIÓN DE CALIDAD EN LAS EMPRESAS DEL SECTOR TURÍSTICO DE COLOMBIA
}

\author{
${ }^{1}$ Diana Leidy Guerrero-Sánchez
}

\section{Resumen}

Objetivo: El objetivo de esta investigación fue analizar si existe o no relación entre el enfoque de calidad y la implementación de un Sistema de Gestión de Calidad en las empresas del sector turístico colombiano, al comprobar, el enfoque de calidad adoptado por las empresas (conformidad o satisfacción al cliente) y la implementación o no del sistema de gestión de la calidad (SGC).

Método: Se aplica un enfoque de investigación cuantitativo de tipo descriptivo-relacional, al realizar un análisis a 134 empresas entre hoteles, restaurantes y agencias de viajes afiliadas y no a las agremiaciones de turismo en Colombia. Los datos miden la relación de las variables propuestas, a través de la técnica análisis multi-grupo y Chi-cuadrado en el programa SPSS.

Originalidad / Relevancia: proponer criterios que determinen el enfoque de la calidad sobre la conceptualización de misma, mediante la ejecución de las practicas organizacionales.

Resultados: Los hallazgos del estudio indican que el enfoque de calidad que se aplica en las empresas turística es independiente de la implantación o no de un sistema de gestión de calidad, dada la naturaleza del sector.

Contribuciones teóricas / metodológicas: (i) Se propone a los gerentes y estrategas de sector turístico, comprender qué elementos de su orientación estratégica están diseñados bajo una perspectiva interna y externa de la empresa, al tener claridad sobre la adopción de un enfoque calidad entendida como conformidad o como satisfacción a las expectativas del cliente; ; (ii) Se recomienda atender aquellos procesos de mejora continua que garantizan el éxito del producto turístico y su competitividad, al prestar servicios personalizados, ofertar paquetes turísticos y actividades complementarias del destino. Finalmente, se sugiere incrementar las investigaciones de corte empírico, basado principalmente en técnicas cuantitativas, particularmente del sector turístico en Colombia en el campo de la calidad, dada la limitada literatura sobre el tema.

Palabras claves: Turismo, calidad, Sistemas de Gestión de Calidad, satisfacción del cliente.

\section{Como referenciar em APA:}

Guerrero-Sánchez, D. L. (2019). Implantación de un sistema de gestión de calidad en la empresas del sector turístico de Colombia. PODIUM Sport, Leisure And Tourism Review, 8(2), 241-252. https://doi.org/10.5585/podium.v8i2.10810

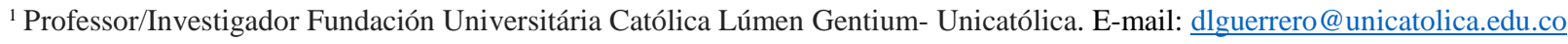
Orcid: https://orcid.org/0000-0002-9365-3371
} 


\title{
IMPLEMENTATION OF A QUALITY MANAGEMENT SYSTEM IN COMPANIES OF COLOMBIA TOURIST SECTOR
}

\begin{abstract}
Objective: The objective of this research was to analyze if does exist or not a relationship between the quality approach and the implementation of the Quality Management System in the companies of the Colombian tourism sector, testing the quality approach adopted by the companies (compliance or customer satisfaction) and the implementation or not of the quality management system (QMS).

Methodology / Approach: A quantitative research approach of descriptive-relational type is applied, analyzing 134 companies between hotels, restaurants and affiliated travel agencies and not to tourism associations in Colombia. The data measure the relationship of the proposed variables, through the multi-group and Chi-square analysis technique in the SPSS program.

Originality / Relevance: Propose criteria to determine the quality approach on the conceptualization of same, by implementing organizational practices.

Main results: The study findings indicate that the quality approach applied in tourism companies is independent of the implementation or not of a quality management system, given the nature of the sector.

Theoretical / methodological contributions: (i) It is proposed to managers and strategists of the tourism sector, to understand what elements of their strategic orientation are designed from an internal and external perspective of the company, having clarity on the adoption of a quality approach understood as compliance or as satisfaction to customer expectations; (ii) It is recommended to attend those processes of continuous improvement that guarantee the success of the tourist product and its competitiveness, by providing personalized services, offering tourist packages and complementary activities of the destination. Finally, it is suggested to increase empirical research, based mainly on quantitative techniques, particularly of the tourism sector in Colombia in the field of quality, given the limited literature about the topic.
\end{abstract}

Keywords: Quality, Tourism, Quality Management Systems, customer satisfaction. 


\section{IMPLEMENTAÇÃO DE UM SISTEMA DE GESTÃO DA QUALIDADE NAS EMPRESAS DO SETOR DE TURISMO DA COLÔMBIA}

\section{Resumo}

Objetivo do estudo: O objetivo desta pesquisa foi analisar se existe ou não relação entre o foco da qualidade e a implementação de um Sistema de Gestão da Qualidade nas empresas do setor turístico da Colômbia, ao verificar o foco da qualidade adotado pelas empresas (conformidade ou satisfação do cliente) e a implementação ou não do sistema de gestão da qualidade (SGQ).

Metodologia/ abordagem: Foi aplicado um foco de pesquisa quantitativa do tipo descritivorelacional, ao analisar 134 empresas entre hotéis, restaurantes e agências de viagens afiliadas e não a associações de turismo na Colômbia. Os dados medem a relação das variáveis propostas, por meio da técnica de análise multi-grupo e qui-quadrado no programa SPSS.

Originalidade / Relevância: Propor critérios que determinem a abordagem da qualidade para sua conceituação, através da execução de práticas organizacionais.

Principais resultados: Os resultados do estudo indicam que o foco da qualidade aplicado nas empresas de turismo é independente da implementação ou não de um sistema de gestão da qualidade, dada a natureza do setor.

Contribuições teóricas / metodológicas: (i) Propõe-se na gerencia e estrategistas do setor do turismo compreender quais elementos de sua orientação estratégica são projetados sob uma perspectiva interna e externa da empresa, com clareza na adoção de um foco de qualidade entendida como conformidade ou satisfação das expectativas do cliente; ; (ii) Recomenda-se atender aos processos de melhoria contínua que garantam o sucesso do produto turístico e sua competitividade, prestando serviços personalizados, oferecer pacotes turísticos e atividades complementares do destino. Por fim, sugere-se aumentar a pesquisa empírica, baseada principalmente em técnicas quantitativas, particularmente do setor de turismo na Colômbia no campo da qualidade, dada a limitação da literatura sobre o assunto.

Palavras-chave: Turismo, qualidade, Sistemas de Gestão da Qualidade, satisfação do cliente. 


\section{Introducción}

Las empresas que interactúan en el sector turístico (hoteles, restaurantes, agencias de viajes, transporte etc.), desarrollan actividades integradas e interrelacionas, dados los espacios y recursos geográficos disponibles en los lugares de destinos y en donde la llegada de visitantes nacionales y extranjeros crece significativamente (GuerreroSánchez, 2018)

De modo que, el término calidad es un concepto clave para entender la competitividad de las organizaciones en los diferentes sectores económicos, y en especial, en el sector turístico. Así, la definición y el dimensionamiento del concepto de calidad ha ido evolucionando y adaptándose a medida que las empresas buscan su aplicación (Camisón, Cruz, y González, 2007), a través de la implantación de sistemas de gestión calidad (SGC), que generen reconocimiento y prestigios en sus actividades comerciales, dadas las limitaciones de competir solamente con precios y en el que el cambio en la percepción de los usuarios es inestable.

Por tanto, se hace imprescindible aprehender del conocimiento acumulado en las dos últimas décadas del Siglo XX y comprender los diferentes conceptos $\mathrm{y}$ enfoques de calidad, ya que la manera de interpretar o concebir la calidad orienta y condiciona el propósito de la dirección (Tena, Llusar y Puig, 2001) y el modo en el que éstas definen e implementan las estrategias de gestión de calidad en la organización. Sin embargo, pese al acotamiento del estudio de la calidad en la literatura especializada, el impacto de esta investigación en el sector turístico colombiano, aun es escaso.

Según Zuñiga-Collazos (2015) es necesario aumentar los estudios en el campo del turismo en Colombia, pues ninguna de las investigaciones analizadas en las publicaciones identificadas en el periodo comprendido entre 2007 y 2013 tiene como tópico la calidad y/o innovación en turismo en dicho país. Por otro lado, se encuentran conceptos que ponen el foco en el interior de la organización y en los componentes de la industria dirigiéndose la mayoría de los esfuerzos, en estudios sobre el sector industrial y en el ámbito de la dirección de operaciones de producción, siendo menor, el enfoque hacia el mercado entendido como grupo de clientes las expectativas por satisfacer de un grupo de visitantes en la condición de turista.

Así pues, las acciones de las empresas de turismo deben responder a las necesidades latentes del turista para proporcionar satisfacción y lealtad de los mismos, ya que una mayor calidad en la prestación de productos turísticos permitirá que los usuarios o turistas perciban experiencias placenteras e incomparables, concibiendo la identidad de los destinos turísticos visitados y apreciando las experiencias vivenciadas en el lugar. Por lo tanto, dicha satisfacción implica que posiblemente repitan su elección o recomienden a otros que visiten el destino, lo que supondrá grandes beneficios económicos y ventajas competitivas para las empresas (León, 2005).

En tal sentido, el objetivo de esta investigación es analizar si existe o no relación entre el enfoque de calidad y la implementación de un Sistema de Gestión de Calidad en las empresas del sector turístico colombiano. Para ello, se analiza el concepto de calidad que las empresas encuestadas adoptan, partiendo de las diferentes perspectivas teóricas que dan fundamento a sus definiciones y que conllevan a la admisión de un determinado enfoque de gestión de calidad "conformidad /satisfacción" y a la implantación o no del SGC. La metodología se evalúa mediante una prueba estadística multigrupo y el análisis chi-cuadrado, en el programa SPSS a una muestra de 134 empresas del sector turístico colombiano.

\section{Marco Teórico}

El buen comportamiento y desempeño del sector turístico contribuye al crecimiento económico y a la generación de empleo en muchos lugares del mundo, por ello, es importante que los países promuevan políticas para fomentar el crecimiento y la competitividad del turismo, promocionando los viajes, invirtiendo en los lugares de destinos, fortaleciendo el recurso humano y generando calidad, innovación y sostenibilidad en el sector (Guerrero-Sánchez, 2018).

Son diversas las corrientes literarias y los autores (De la Ballina y Peláez (2016; Camisón et al. 2007; Tena et al. 2002; Cruz 2001), que contribuyen a solucionar y a establecer mecanismos y métodos que sustentan la aplicación de la calidad en los diferentes sectores y tipos de organizaciones, así como las situaciones cambiantes que acontecen en las mismas Sin embargo, el carácter subjetivo y abstracto de la calidad (Paladini, 2011), aún es materia de diversas aplicaciones e interpretaciones en el contexto científico y en las empresas. 
En el sector turístico según De la Ballina Ballina y Peláez (2016) se pueden diferenciar tres señales de calidad: las llamadas denominaciones de origen relacionadas a las marcas regionales, las certificaciones basadas en una norma y algún premio de calidad de prestigio sectorial. De modo que, el papel de un estándar de calidad genera ventajas competitivas para las empresas hoteleras y turísticas, ya que tal como indica De la Ballina y Peláez (2016), citando a los siguientes autores, también pueden presentarse otras situaciones como: las presiones de los clientes (Chan, 2008), de los proveedores (King, 2005) o el acceso a nuevos mercados turísticos (Sampaio, Saraiva y Guimaraes, 2009).

Lo cierto es, que los beneficios y los resultados que generan la adopción de un enfoque de gestión o la implementación de un SGC en las organizaciones pasa por reconocer el mejoramiento continuo y el mejor desempeño de las empresas al interior de sus procesos y la generación de ventajas competitivas en el mercado, desde un enfoque externo integral que permite tener una perspectiva global en las estrategias de las empresas.

Algunos estudios han direccionado sus esfuerzos hacia la concepción de que la calidad es una filosofía de dirección o una función directiva más (Camisón, Cruz y Gonzales, 2007; Bernal y Alesón, 2012), un cambio de paradigma que adoptan las organizaciones (Ishikawa, 1954; Brocka y Brocka, 1992), una opción estratégica (Garvin, 1988; Stahl y Grigsby, 1997; Brickley, Smith y Zimmerman, 2004) o simplemente una gestión integral (Cuatrecasas, 2005) que está diseñada para mejorar continuamente el desempeño, considerando las necesidades de todas las partes interesadas de la organización (GuerraVázquez, 2007).

En tal sentido, los hoteles, restaurantes y agencias de viajes obtienen ventajas competitivas desde dos perspectivas básicamente, una estrategia orientada en el establecimiento de precios acorde al servicio prestado y a la gestión de bajo costo a través del descuento; y la otra, basada en direccionar la calidad al ofrecer a los clientes una experiencia ideal para lograr la lealtad de los mismos (Ooi, 2014). De modo que:

Ante la circulación del flujo de personas a diversos destinos turísticos, las empresas necesitan resultados competitivos de sus estrategias, para realizar una gestión comunicativa eficiente del lugar turístico, frente a la toma de decisiones relevantes del turista, al momento de su elección, debido al abanico de opciones que tiene este para adquirir un producto o servicio (Guerrero-Sánchez, 2018, p. 352).

Po otra parte, para Osman y Sentosa (2013) existe una relación significativa entre certificación de calidad y la lealtad del cliente, en tal sentido, promover la calidad mediante la certificación de las ISO 9000 y las Normas Técnicas Sectoriales de Calidad en los operadores del sector turístico, está dada por la implantación de estrategias que mejoren de la calidad en la prestación de los servicios turísticos; y en donde la adhesión a un estándar de calidad, determinada por la categorización de un hotel restaurante o agencia de viajes permite la obtención de mejores resultados, el incremento de la rentabilidad y la competitividad en las empresas que los implantan (Alonso y Rodríguez, 2011).

Desde esta óptica, la calidad puede ser vista como una aproximación de lo que sería la implementación de un SGC en las organizaciones y en donde los diferentes conceptos de calidad proporcionan diferentes orientaciones para su funcionamiento. Ello pasa por reconocer los diferentes conceptos de calidad y la orientación de un respectivo enfoque de gestión, considerados en las siguientes definiciones:

la calidad como conformidad que está fundamentada con base a unas especificaciones y el cumplimiento a unos requisitos (Garvin,1988); la calidad como adecuación al uso, que es entendida como la eliminación de las deficiencias, haciendo el producto apto para su uso (Crosby,1989; Feigenbaum, 1991; Juran, 1993) y la calidad como evitar las perdidas, en busca de minimizar la variabilidad en los procesos y la producción (Taguchi y Elsayed, 1989).

Por otro lado, la calidad entendida como valor, parte de la premisa de la relación coste/beneficio determinado por el precio que esté dispuesto a pagar el usuario o turista (Feigenbaum, 1961; García, 2001) y, finalmente, la calidad como satisfacción de las expectativas del cliente, que concibe la calidad como el nivel de satisfacción de las expectativas de los usuarios o consumidores (Parasuraman, Zeithaml y Berry, 1985; Feigenbaum, 1991; García, 2001).

Así pues, los conceptos de la calidad presentados actualmente, siguen siendo utilizados en la literatura sobre el tema, siendo un legado de los "Gurús de la Calidad" para orientar y esclarecer su función como enfoques de gestión y su aplicación en los diferentes contextos.

La Figura 1, muestra el modelo de investigación, en donde las empresas que aplican los conceptos de conformidad, adecuación y evitar fallos se 
consolidan en un sólo enfoque llamado, "conformidad con las especificaciones", siguiendo una orientación de gestión hacia la inspección de los procesos y control de la calidad Total (CCT), al seguir un enfoque interno y reactivo en la organización. Por otro lado, aquellas empresas que adoptan un enfoque de "satisfacción de las expectativas del cliente", indican tanto un concepto de satisfacción como de calidad entendida como valor, redirigiendo por tanto sus esfuerzos a consolidar un enfoque de Gestión de la Calidad Total (GCT), mediante una perspectiva externa de la organización orientada hacia el cliente y el mercado. La presente clasificación se adaptó, conforme a los conceptos de calidad planteados por Escrig (1998) y Cruz (2001) y que reposan en el despliegue de un enfoque del SGC.

Figura. 1 - Los conceptos de calidad en función del enfoque de gestión de calidad

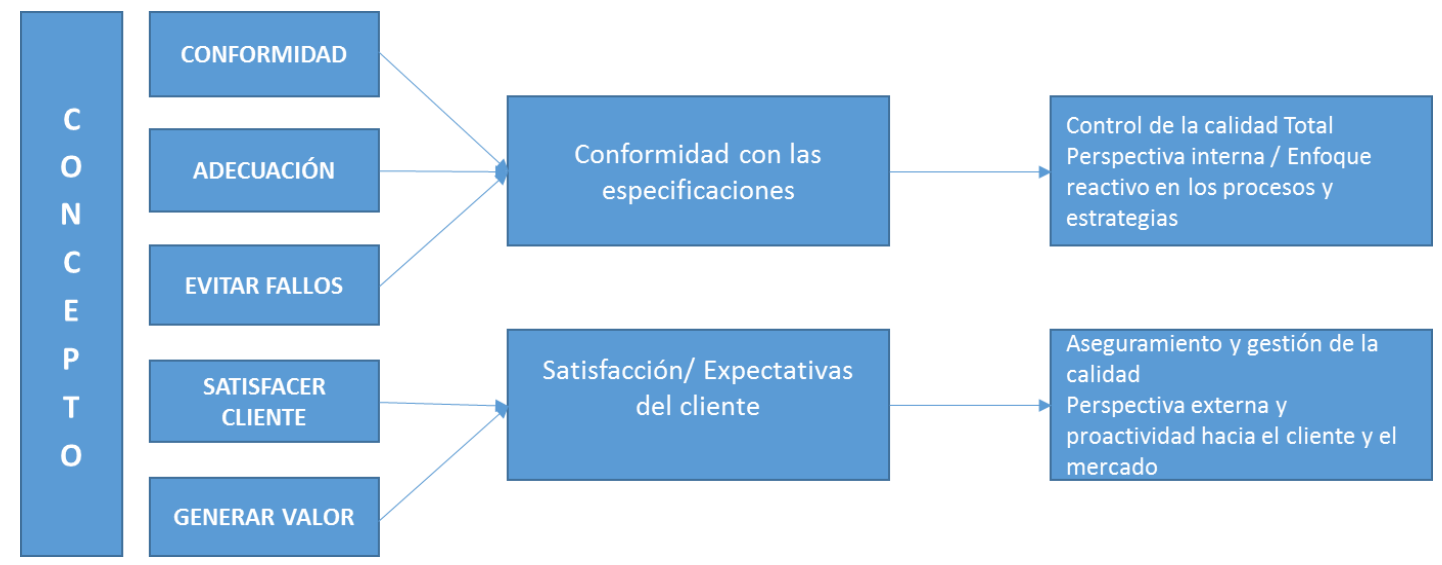

Fuente: Elaboración propia

Por lo anterior, dependiendo del modo en el que cada organización define su concepto de calidad, ésta adoptará un enfoque u otro para la implementación del SGC en las empresas. Así como, el concepto de calidad acuñado por la organización será expresión del propósito de la organización.

\section{Metodología}

El modelo teórico presentado permitió delimitar el objeto de estudio de esta investigación, al cuestionarse ¿Cuál es la relación entre el enfoque de calidad y la implementación o no de un Sistema de Gestión de Calidad en las empresas del sector turístico colombiano?

Para describir la metodología de la investigación, se hizo necesario revisar el contexto del sector turístico en Colombia. El trabajo de campo se inició con la determinación y estratificación de la muestra de Micro - pequeñas y medianas empresas del sector turismo inscritas a la Cámara de Comercio de Colombia, en donde la participación por ubicación geografía se generó entre las principales regiones del país, distinguiendo sus principales ciudades, que para el caso de Valle del cuaca, Cali obtuvo una participación del 61\%, Cundinamarca-Bogotá un $15 \%$, Atlántico-Barranquilla un $11 \%$ y finalmente otras regiones cerca del $30 \%$ (Tabla 2). La población en el ámbito de estudio fue de 1000 empresas, entre hoteles, restaurantes, agencias de viajes, entre otras (tabla 1), en donde la muestra válida del estudio fue de 134 organizaciones con un error muestral $8 \%$ y un nivel de confianza del $95 \%(\mathrm{Z}=1,96)$, para un $\mathrm{p}=\mathrm{q}=0,5$.

El cuestionario se aplicó a gerentes, directores generales o responsables de calidad. Con relación al tratamiento de los datos, las escalas fueron traducidas y adecuadas al léxico del sujeto de aplicación de las encuestas, se utilizó una escala tipo Likert de 5 puntos, sobre el concepto de calidad como enfoque de gestión (Escrig-Tena y Bou-Llusar, 2002); y preguntas dicotómicas con relación a la implantación del SGC (Ver anexo 1).

Por otro lado, el tratamiento de los datos y el análisis estadístico se realizó a través del programa SPSS, para medir la relación de las variables propuestas, a través del análisis multi-grupo y el Chicuadrado de Pearson, en las empresas objeto de estudio. 


\section{Resultados}

El perfil de las empresas encuestas, se describió a partir de aspectos descriptivo de la muestra que pasaron por determinar el tipo de actividad dentro del sector turístico entre hoteles, restaurantes y agencias de viajes. En donde de las 1000 empresas encuestadas vía online se obtuvieron 134 contestaciones, lo que significó una tasa de respuesta del $13.4 \%$, con mayor representación de los hoteles y restaurantes, en un $40 \%$ y $29 \%$ respectivamente, siendo cerca del $70 \%$ del total (Tabla 1).

Tabla 1 - Actividad de las empresas

\begin{tabular}{c|c|c}
\hline Actividad & Número de empresas & \% empresas \\
\hline Hoteles & 54 & 40,3 \\
\hline Restaurantes & 39 & 29,4 \\
\hline Agencias de viajes & 22 & 16.4 \\
\hline Otras & 19 & 13,9 \\
\hline Total & 134 & 100,0 \\
\hline
\end{tabular}

Fuente: Elaboración propia

Para el caso de la ubicación geográfica de las empresas se obtuvieron datos de las principales ciudades del país, siendo de mayor representación
Cali con un $45 \%$, seguido de Bogotá y Barranquilla con un $19 \%$ y $11 \%$ respectivamente (Tabla 2 ).

Tabla 2 - Ubicación geográfica de las empresas

\begin{tabular}{c|c|c}
\hline Ciudad & Número de empresas & \% empresas \\
\hline Cali & 61 & 45,5 \\
\hline Bogotá & 26 & 19,4 \\
\hline Barranquilla & 15 & 11,2 \\
Medellín & 11 & 8,2 \\
\hline Otras & 21 & 15,7 \\
Total & 134 & 100.0 \\
\hline
\end{tabular}

Fuente: Elaboración propia

Los resultados de este estudio indican que se evidencia que las empresas adoptan un concepto de calidad desde dos enfoques de gestión, uno hacia la orientación a la satisfacción de las expectativas del cliente, con una visión hacia el dinamismo del mercado y el otro, hacia la calidad entendida como conformidad a unas especificaciones dado por un enfoque interno reactivo para la gestión, en correspondencia al enfoque del aseguramiento de la calidad aplicado por las mismas.

Los resultados del análisis descriptivo muestran que cerca del $57.5 \%$ de las empresas encuestadas aplican un concepto de calidad enfocado hacia la satisfacción de las expectativas del cliente, es decir 77 empresas; seguidamente, un $20 \%$ adopta el concepto de la calidad entendida como valor, que es aplicado por 27 empresas; y los demás conceptos, suman el $22.5 \%$, y son amparados por empresas que se identifican con un concepto de conformidad con las especificaciones, la adecuación para el uso y la detención de fallos en sus procesos organizacionales (Tabla 3). 
Tabla 3 - Concepto de calidad que se aplica en la empresa

\begin{tabular}{c|c|c}
\hline Concepto de Calidad & Número de empresas & \% empresas \\
\hline $\begin{array}{c}\text { Que el producto/servicios sea conforme } \\
\text { a las especificaciones }\end{array}$ & 14 & 10,4 \\
\hline Adecuación para el uso. & 14 & 10,4 \\
\hline Satisfacer las expectativas del cliente. & 77 & 57,5 \\
\hline Proporcionar valor al cliente & 27 & 20,2 \\
\hline Evitar fallos & 2 & 1,5 \\
\hline Total & 134 & 100,0 \\
\hline
\end{tabular}

Fuente: Elaboración propia

Con relación a los hallazgos de la medición del enfoque de calidad adoptado por las empresas, el análisis multigrupo arrojó que el grupo 1 está formado por 30 empresas que dijeron, adoptar un concepto de calidad entendida como conformidad con las especificaciones y el grupo 2 está formado por 104 empresas que señalaron que, adoptar la calidad entendida como satisfacer las expectativas de los clientes o calidad como valor (Tabla 4).

Tabla 4 - Agrupación de las empresas en función del enfoque de calidad

\begin{tabular}{|c|c|c|}
\hline Enfoque de calidad adoptado & Número de empresas & \% empresas \\
\hline Empresas Concepto de Conformidad & 30 & 22,4 \\
\hline Empresas Concepto de Enfoque al cliente & 104 & 77,6 \\
\hline Total & 134 & 100,0 \\
\hline
\end{tabular}

Fuente: Elaboración propia. Resultados SPSS

De manera que, el enfoque de calidad seguido por las empresas con enfoque al cliente está determinado por un total de 104 empresas que conciben la calidad como la búsqueda en la satisfacción de las expectativas del turista y la generación de valor a un precio dado en la prestación de los productos turísticos, siendo en términos porcentuales, un $77,6 \%$. Por otro lado, se encuentran 30 las empresas que entienden la calidad como un enfoque de conformidad con las especificaciones, siendo un $22,4 \%$ de la muestra total, en donde dichas empresas están orientadas al control de los procesos y el evitar fallos en la prestación de los servicios turísticos, sin embargo, sus estrategias, no están orientadas a las tendencias del mercado. La representación porcentual gráfica de dicha participación se puede apreciar en la figura 2.

Figura 2 - Distribución de la muestra en función del enfoque de gestión de calidad

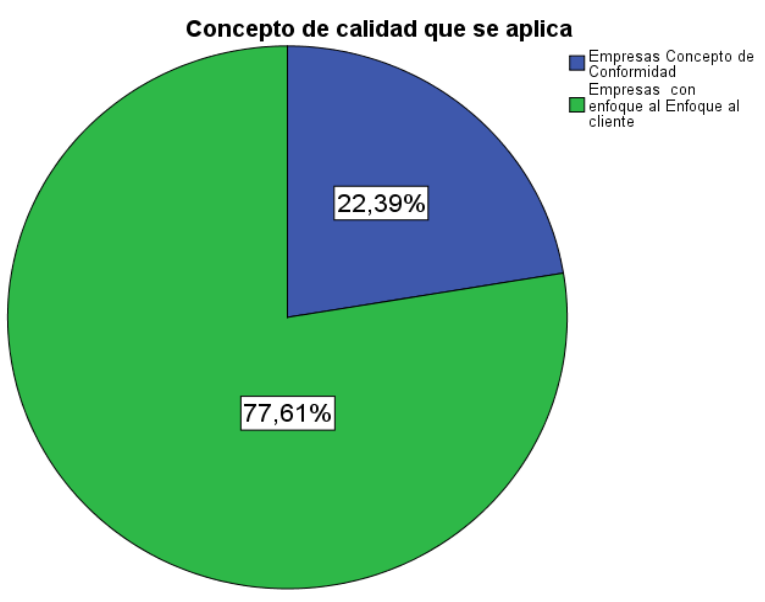

Fuente: Elaboración propia. Resultados SPSS 
Por otro lado, el análisis en los resultados de la relación entre el concepto de calidad adoptado por las empresas (conformidad o satisfacción al cliente) y la implementación o no del sistema de gestión de la calidad, evaluado mediante la prueba $J i$-cuadrado de Pearson, arrojo un valor $\mathrm{X}^{2}=4,562$, y un $p$ valor de 0,335 . A raíz de estos resultados, se puede inferir que no existe una relación significativa entre el enfoque de calidad y la implantación del SGC, lo que implica que el enfoque de calidad que se aplica en cada empresa es independiente de la implantación o no del sistema de gestión en las empresas turísticas de Colombia (Tabla 5).

Tabla 5 - Concepto de calidad adoptado por las empresas que han implantado, o no han implantado el SGC.

\begin{tabular}{|c|c|c|c|c|}
\hline \multicolumn{2}{|l|}{$\begin{array}{l}\text { Empresas que han } \\
\text { Implantado SGC }\end{array}$} & \multicolumn{3}{|c|}{ Concepto de Calidad } \\
\hline & & \multirow{2}{*}{$\begin{array}{l}\text { Conformidad } \\
14\end{array}$} & $\begin{array}{l}\text { Enfoque a al } \\
\text { Cliente }\end{array}$ & Total \\
\hline & Recuento & & 39 & 53 \\
\hline NO & $\%$ de empresas & $46.7 \%$ & $37.5 \%$ & $39,6 \%$ \\
\hline \multirow[t]{4}{*}{$\overline{\text { SI }}$} & Recuento & 16 & 65 & 81 \\
\hline & $\%$ de empresas & $53.3 \%$ & $62,5 \%$ & $60,4 \%$ \\
\hline & & 30 & 104 & 134 \\
\hline & & $100 \%$ & $100 \%$ & 100 \\
\hline \multicolumn{5}{|c|}{ Nivel de significación $(\alpha)=0,05$} \\
\hline \multicolumn{5}{|c|}{$\chi^{2}=4,562$} \\
\hline$P$ value $=0,335$ & & & & \\
\hline
\end{tabular}

Los resultados obtenidos comprueban que el $37.50 \%$ de las empresas que no han implantado un SGC adoptan un enfoque hacia la satisfacción las necesidades, frente al $62,50 \%$ de las que sí lo han implantado. Por otra parte, si se analiza el concepto de calidad con relación a la conformidad, la diferencia es aún menos significativa, al considerar que el $53.33 \%$ de las empresas que implantado un SGC han adoptado un concepto de calidad de conformidad frente al 46,67\% de aquellas empresas que no han implantado ningún sistema, demostrándose que no existe una relación significativa entre las variables de estudio.

\section{Discusiones}

A la luz de los hallazgos de esta investigación, se expone que la relación entre el enfoque de calidad adoptado por las empresas (conformidad y satisfacción al cliente) y la implementación o no de un sistema de gestión en las empresas del sector turístico colombiano, resulta ser no significativa, al existir total independencia en el análisis de la relación entre las variables estudiadas.

En relación con los resultados obtenidos, se establece que las empresas turísticas de Colombia, que adoptan un enfoque de calidad entendido como conformidad, tienen una orientación interna reactiva, incidiendo en el cumplimiento a unos requisitos técnicos tales como las normas ISO o alguna norma técnica sectorial de turismo. Lo que establece que independientemente del enfoque de adopción "Conformidad/satisfacción del cliente" asumido por las empresas turísticas, estas pueden o no asumir la implementación de un SGC, al no interferir ninguna relación significativa.

Sin embargo, en conformidad con las evidencias empíricas el estudio de Cruz-Ros (2001), las orientaciones hacia la adopción de un sistema de GC y su implantación, permiten obtener un mejor desempeño organizativo, que aquellas empresas que aplican orientaciones hacia el aseguramiento de calidad, dada las competencias distintivas en dichos enfoques.

Por lo cual, el hecho de adoptar una concepción de calidad en las empresas, conlleva a que estas realicen prácticas organizativas, que necesariamente operacionalicen la calidad en sus procesos de prestación de servicios. Pues tal como afirman Camisón, Bou y Roca, (2000) las prácticas para cumplir con la calidad se convierten en algo usual con independencia de la existencia de un enfoque de calidad adoptado.

Así, en el contexto del turismo el enfoque de conformidad está dado, por la incorporación de 
procesos técnicos tradicionales necesarios para que el usuario o turista perciba y experimente las características representativas del lugar visitado, mediante documentos informativos (Fichas, audios, folletos, etc.), sin presentarse mejoras significativas e innovaciones, así como tampoco, ajustes importantes en la prestación de los productos turísticos (instalaciones, incorporación de tecnología, nuevos planes turísticos, etc.), conforme a las necesidades del visitante, sin embargo, según los resultados de esta investigación estas orientaciones no eximen a las empresas turísticas de tomar la decisión de implementar un SGC total, para la visualización de experiencias significativas para la oferta de productos turísticos y las tendencias del mercado.

Frente a lo expuesto, el dejar de estar sometido a una actitud meramente contemplativa y pasiva, aumentando la competitividad, en donde la calidad del servicio determine las condiciones de seguridad, confort, accesibilidad, etc., en la prestación de la calidad de cualquier servicio turístico, garantiza una correcta gestión de los recursos, para así transformarlos en productos competitivos que garanticen una óptima oferta de los productos turísticos (León, 2005)

\section{Conclusiones}

Se puedo comprobar que el enfoque de calidad que se aplica en cada empresa es independiente de la implantación o no del sistema de gestión de calidad y que con independencia del enfoque de calidad adoptado, los sistemas de GC (El abanico de normas ISO y las Norma Técnicas Sectoriales de Turismo) ponen el énfasis en capacitar y empoderar a los miembros de la organización ya que, los directivos deben ser conscientes de las implicaciones de adoptar un concepto de calidad que orienta la intencionalidad de la dirección y bajo el cual se desarrollan un conjunto de estrategias, siendo dependientes del enfoque del SGC que se lleve a cabo.

Bajo estas consideraciones, para mantenerse en la dinamismo actual, se hace indispensable apóstale a las tendencias modernistas de la certificación a través de la implementación de los Sistemas de Calidad, ya que como bien lo plantean varias investigaciones las empresas que han implantando sistemas de aseguramiento, de calidad, vislumbran una planificación a futuro para implantar un sistema de

\section{Bibliografía}

Alonso, M. \& Rodríguez, J. 2011. “Organizational behavior and strategies in the adoption of certified gestión de la calidad total que presume gestiones más sólidas para el logro de ventajas competitivas (Ho, 1994; Wenmoth y Dobbin, 1994; Weston, 1995; Lee, Leung y Chan, 1999).

Por lo tanto, suman importancia aquellos procesos de mejora continua que garantizan el éxito del producto turístico y su competitividad, al prestar servicios personalizados, ofertar paquetes turísticos y actividades complementarias del destino, promoviendo ofertas d productos innovadores acorde a los cambios y tendencias que se presentan en el entorno y mediados por el uso de las Tecnologías de la información y la comunicación (TICs), como insignia de los nuevos escenarios que generan lealtad en la sociedad.

Esta investigación permitirá a los gerentes y estrategas de sector turístico, comprender qué elementos de su orientación estratégica están diseñados bajo una perspectiva interna y externa de la empresa, al tener claridad sobre la adopción de un enfoque calidad entendida como conformidad o como satisfacción a las expectativas del cliente. De modo que, dichos aspectos del SGC les permitirán mejorar continuamente, generar ventajas competitivas y buscar la satisfacción de los usuarios vía diferenciación en los productos y servicios turísticos que ofrecen.

De tal manera, que las expectativas u objetivos para implantar un sistema de aseguramiento de la calidad son la mejora de los resultados económicos, la ampliación y mantenimiento de la cartera de clientes y la mejora de la eficiencia (Martínez, Balbastre, Escribá, González y Pardo, 1999), ya que la implantación de una estándar calidad debe ser la base de un proceso de estandarización progresiva sobre la cual, se aumenten los resultados económicos y comerciales, ya sea con las marcas regionales aumentando la fuerza comercializadora de los destinos (De la Ballina y Peláez, 2016).

Finalmente, se sugiere incrementar las investigaciones de corte empírico, basado principalmente en técnicas cuantitativas, particularmente del sector turístico en Colombia en el campo de la calidad, dada la imitada literatura sobre el tema. Y, para futuros trabajos, será necesario establecer criterios entre los diferentes tipos de empresas del sector, en función de los principios o prácticas de gestión de calidad que les permiten satisfacer las necesidades y expectativas de los usuarios, vía diferenciación con la finalidad de promover lealtad de los visitantes en los destinos turísticos.

management systems". Journal of Cleaner Production, 19 , pp. 1455- 1463.

Bernal, J., \& Alesón, M. (2012). Naturaleza, significado y contenido de la gestión de la calidad total. 
Revista Icade. Revista de las Facultades de Derecho y Ciencias Económicas y Empresariales, (73), 225-240.

Brickley, J., Smith, C., \& Zimmerman, J. (2004). Managerial Economics and organizational architecture. 3rd. edition. Nova Iorque: McGrawHill/Irwin.

Brocka, B., \& Brocka, M. (1992). Quality management: Implementing the best ideas of the masters. McGraw-Hill.

Camisón, C., J.C. Bou y V. Roca (2000): "Enfoque de gestión de calidad y desempeño organizativo: el rol contingente de la estructura de propiedad y control y del estilo directivo". Universidad Ja ume I, Unidad de Investigación en Estrategia, gestión del conocimiento y aprendizaje organizativo, Documento de Trabajo, Castellón

Camisón, C., Cruz, S., \& González, T. (2007). Gestión de la calidad: conceptos, enfoques, modelos y sistemas. Prentice Hill.

Chan, E. S. (2008). Barriers to EMS in the hotel industry. International Journal of Hospitality Management, 27(2), 187-196.

Crosby, P. (1989). How goes the quality revolution? Journal for Quality and Participation, 12(1), 28-31.

Cruz-Ros, S. (2001). Relación entre el enfoque de gestión de la calidad y el desempeño organizativo. Una aproximación desde la perspectiva basada en los recursos. Universitat de València.

Cuatrecasas, 11. (2005): Gestión Integral de la Calidad: Implantación, Control y Certificación. Barcelona: Ediciones Gestión 2000.

De la Ballina Ballina, F. J., \& Peláez, L. V. (2016). Los efectos de la combinación de estándares de calidad sobre los resultados empresariales en el sector hotelero. Universia Business Review, (52).

Escrig Tena, A. (2001). Efectos de la dirección de calidad en los resultados: el papel mediador de las competencias distintivas. Universitat Jaume I.

Feigenbaum, A. (1961): Total Quality Control. New York: McGraw-Hill.

Feigenbaum, A. (1991), Total Quality Control, 3rd ed., McGraw-Hill, New York, NY.
García, R. (2001). El concepto de calidad y su aplicación en Medicina. Revista médica de Chile, 129(7), 825-826.

Garvin, D. (1988). Managing quality: The strategic and competitive edge.

Guerra -Vázquez, L. (2007). Propuesta del diseño del sistema de gestión de la calidad para el servicio de adiestramiento e información científico-técnica en la oficina de normalización de camagüey y las vías de su implementación, Universidad de Camagüey.

Guerrero-Sánchez, D. (2018). La comunicación y la promoción como estrategias del marketing turístico Aranzadi (Ed.), Las fuentes de información turística en foco (pp.349 -367). España: Thomson Reuters

Ho, S. (1994): "Is the ISO 9000 Series for Total Quality Management?". International Journal of Quality \& Reliability Management, 11 (9), 74-89.

Ishikawa, K. (1954), Hinshitsu Kanri Nyumon. JUSE Press Ltd., Tokio. La 3.a edición se publicó en 1988. Edición inglesa: Introduction to quality control.

Juran, J. (1993): "Why quality initiatives fail?". Journal of Business Strategy, 14 (5), 35-38.

King, A. A., Lenox, M. J., \& Terlaak, A. (2005). The strategic use of decentralized institutions: Exploring certification with the ISO 14001 management standard. Academy of management journal, 48(6), 1091-1106.

Lee, T.Y., H.K.N. Leung y K.C.C. Chan (1999): "Improving quality management on the basis of ISO 9000”. The TQM Magazine, 11 (2), 88-94.

León, J. F. C. (2005). La Calidad como herramienta de gestión del Turismo Cultural. Revista de turismo e patrimonio cultural 3 (1), 143.

Martínez, C., F. Balbastre, M.A. Escribá, T. González y M. Pardo (1999): "Análisis de la implantación de un sistema de aseguramiento de calidad en base a normas ISO 9000". IX Congreso Nacional de ACEDE, Septiembre, Burgos. CD

Ooi, K.B. (2014). TQM: ¿A facilitator to enhance knowledge management? A structural analysis. Expert Systems with Applications, 41(11), 5167-517.

Osman, Z., \& Sentosa, I. (2013). Mediating effect of customer satisfaction on service quality and customer loyalty relationship in Malaysian rural tourism. 
Paladini, E. (2011). Gestão da Qualidade: teoria e prática. São Paulo: Atlas.

Parasuraman, A., Zeithaml V. y Berry L. (1985): “A conceptual model of service quality and its implications for future research". Journal of Marketing, 49 (3), 4150 .

Sampaio, P., Saraiva, P. Y Guimaraes, A. (2009). ISO 9001 certification research: questions, answers and approaches". International Journal of Quality and Reliability Management, 26 (1), 38-58

Stahl, M. \& Grigsby, D. (1997). Strategic management: total quality and global competition. Wiley.

Taguchi, G. y Elsayed, E. (1989), Quality Engineering in Productions Systems. McGraw-Hill, Nueva York. The International Journal of Quality Science, 3 (4), 328-347.

Tena, A., Llusar, J, \& Puig, V. (2001). Measuring the relationship between total quality management and sustainable competitive advantage: A resource-based view. Total quality management, 12(7-8), 932-938.

Tena, A. B. E., \& Llusar, J. C. B. (2002). Desarrollo y validación de un instrumento de medida de la dirección de la calidad: una propuesta de mejora. Investigaciones Europeas de Dirección y Economía de la Empresa, 8(1), 151-176.

Wenmoth, B. y D. Dobbin (1994): "Experience with implementing ISO 9000". Asia Pacific Journal of Quality Management, 3 (3), 9-27.

Weston, F.C. (1995): "What do managers really think about registration process?". Quality Progress, 28 (10), 67-73.

Zuñiga-Collazos, A. (2015). Análisis de la investigación y desarrollo turístico en Colombia. Revista espacios| 36 (18). 